\title{
CONSIDERACIONES PRELIMINARES
}

\author{
MARIA JOSE ESCARTIN CAPARROS
}

Profesora de Trabajo Social EUTS de Alicante

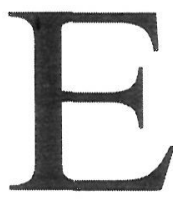

n el momento de programar y estructurar el primer número de nuestra revista de Trabajo Social, cuya publicación se acordó a comienzos del presente curso académico 1991-1992, los responsables de esta tarea hemos pensado que la familia podía constituir, por razones obvias, un tema monográfico de gran interés para todos los profesionales que realizan su actividad en este campo.

Conscientes, por otra parte, de la multiplicidad de aspectos y problemas que inciden en este ámbito y de su complejidad, entendíamos que era preciso delimitar el campo de nuestros análisis y estudios, teniendo en cuenta fundamentalmente los fines específicos de nuestra labor profesional en la comunidad ${ }^{1}$. Ello nos ha conducido a insistir de forma preferente en aspectos concretos muy próximos a nuestro entorno social y cultural. Todas y cada una de las colaboraciones que se contienen en este número inaugural responden, de un modo u otro, a esta convicción común básica.

Pero al abordar y desarrollar los diversos problemas que son objeto de estudio está presente, lógicamente, una conciencia más amplia y abarcadora de la situación actual de la familia y de sus perspectivas de futuro en nuestras sociedades occidentales. En este sentido, se tienen muy en cuenta las investigaciones actuales a propósito de la transformación de las relaciones familiares, cuya comprensión y valoración exige análisis relacionados, por ejemplo, con el cambio social, la identidad individual y colectiva, el proceso de separación entre las esferas familiar y extrafamiliar, las condiciones económicas y de trabajo, la incidencia de las ideologías o la intensidad de las relaciones entre los diversos pueblos y culturas ${ }^{2}$.

Prolongando esta línea de reflexión, nos proponemos seguir aten-

${ }^{1}$ En este sentido, entiendo que es muy positivo el esfuerzo de $\mathrm{M}$. Whan en resaltar la importancia de lo que considera decisivo en nuestro trabajo: «to retrieve a sense of social work practice which recognises it as a form of practical, moral engagement and not primarily as a matter of technique» (On the nature of Practice, British Journal Of Social Work, 16, 1986 pág. 243).

${ }^{2}$ A propósito de los cambios en la estructura y funcionamiento de la familia durante las últimas décadas, cf. L. Cseh-Szombathy, la modélisation des interactions société-famille, Rév. Int. Sc. Soc., núm. 126, 1990, págs. 492 y sigs. 
tamente los estudios y proyectos que están haciéndose a propósito del futuro de la familia y su configuración, especialmente en los países de Occidente. El carácter interdisciplinar que preside esta labor hace posible el acceso a una comprensión más profunda del proceso que está en marcha, teniendo en cuenta las aportaciones convergentes de la antropología, la sociología, el derecho, la demografía, la economía o la política ${ }^{3}$.

Refiriéndose a las conclusiones de los grupos de trabajo que coordina la Unesco, D. Behnam subraya el hecho de que aunque la institución familiar sigue vigente, es innegable la diversidad estructural y funcional de los sistemas familiares, de modo que no puede hablarse de ninguna teoría general o modelo universal. Por otra parte, se mantiene y acentúa el papel del Estado y la necesidad de su intervención, que ha de traducirse en políticas sociales y demográficas, así como en reformas jurídicas. Finalmente, se advierte la conveniencia de que las múltiples formas de intervención sectorial (niños, ancianos, jóvenes, mujeres, grupos marginados) se integren y armonicen dentro del marco familiar. En cuanto a los cambios y transformaciones que van teniendo lugar en la familia, dentro del ámbito de nuestras sociedades industriales, se hace referencia a algunas tendencias dominantes como las relaciones extramatrimoniales (diversas formas de cohabitación) y la posibilidad de renovación de la familia conyugal por influencia del descenso de la mortalidad o el progreso de los recursos médico-quirúrgicos (procreación artificial), así como a la aparición de formas alternativas de familia ${ }^{4}$.

Nuestro propósito fundamenal consiste en mantenernos próximos a las nuevas orientaciones y tendencias que van surgiendo tanto en relación con planteamientos teóricos como en referencia a los nuevos modos de afrontar los problemas concretos de la familia. Y somos conscientes de que este empeño no es posible sin el esfuerzo permanente por actualizar los conocimientos, enriquecer el ámbito de las experiencias, perfeccionar los métodos de análisis, de información y de intervención en los conflictos.

Un ejemplo valioso, entre otros muchos, de la importancia de esta

${ }^{3}$ En relación con los cambios que han tenido lugar en el matrimonio, y la familia, son orientadoras las consideraciones que hace W. Goode en su trabajo World Revolution and Family Patterns, en el volumen "The Future as a Social Problem», Santa Mónica, California, 1977, págs. 158 y sigs.

${ }^{4} \mathrm{D}$. Behnam, Une réflexion internationale sur l'avenir de la familie, Rév. Int. Sc. Soc., núm. 126, 1990, págs. 604 y sigs. El autor concluye subrayando el hecho de que "pour mieux saisir la réalité des divers systèmes familiaux, il faut prendre en considération les différences socioculturelles», pág. 608. Según él, la búsqueda de un modelo universal ya no es posible, por lo que una característica de las futuras investigaciones será precisamente la toma en consideración de la diversidad de la familia. 
actitud permanente de búsqueda y creatividad se hace patente hoy en el ámbito de la terapia familiar, a través de la revisión de presupuestos considerados antes básicos, de la ampliación de las formas de intervención y de la búsqueda de nuevos modelos de interacción. Ilustran este sentido crítico y de autoexigencia algunas de las ideas que expone Peggy Papp a propósito de los conceptos y modelos sobre los que se basa la terapia familiar. Aunque inicialmente contribuyeron a ampliar la visión sobre los problemas humanos y a encontrar nuevas formas de tratarlos, constata el hecho de «ahora hemos congelado esos conceptos hasta el punto en el que realmente siento que limitan nuestro campo de visión» ${ }^{5}$. De ahí la necesidad de cuestionar, por ejemplo, el modelo cibernético en el que se basan los conceptos sistémicos, considerándolo excesivamente abstracto y estático como para poder ponerse a la altura de todos los procesos complejos que tienen lugar en las interacciones familiares ${ }^{6}$.

Teniendo presente el contexto socio-histórico en que realizamos nuestras actividades profesionales, es indispensable llegar a tener una visión precisa del alcance real de los cambios que han venido experimentando la institución familiar en nuestro país a lo largo de las últimas décadas, para entender su situación real en las condiciones actuales de vida propias de la llamada «sociedad compleja» ${ }^{7}$.

Piénsese, por ejemplo, en el significado que ha tenido durante ese tiempo la reducción progresiva del tamaño medio de la familia, determinada en notable medida por orientaciones valorativas tendentes a hacer posible la adaptación a las pautas dominantes del desarrollo socio-económico ${ }^{8}$. A ello se añaden las consecuencias que se derivan del trabajo de la mujer fuera del hogar, de la creciente indiferenciación de los roles conyugales y del paso a la nueva forma de autoridad «compartida y democrática» ${ }^{9}$ que es característica de esta etapa de transición en la vida española.

5 Peggy Papp, Carta a Salvador Minuchin, en el vol. Col. «Cambio familiar: Desarrollos de modelos», Gedisa, Ed., Barcelona, 1988, pág. 219.

6 IB., págs. 219 y sigs.; más adelante, refiriéndose al concepto de homeostasis lo describe como demasiado mecánico, fijo y simplista. Su conclusión es que «cualquier concepto que no tenga en cuenta el efecto del tiempo, los acontecimientos repentinos e inesperados y las desviaciones impredecibles de la conducta humana no es sólo inadecuado sino que es terapéuticamente limitado. Después de todo, las familias han estado cambiando durante miles de años, aun cuando no había terapeutas familiares para ayudarlas. Si el concepto de homeostasis fuera exacto, no existiría el cambio espontáneo», pág. 220.

${ }^{7}$ Para entender los múltiples factores que inciden en la actual sociedad compleja, cf. varios: La sfida della complessità, Feltrinelli, Milán, 1987.

8 J. L. Recio Adrados, Los cambios en la familia española, Doc. Soc. núm. 50 , 1983, págs. 89 y sigs.

${ }_{9}$ S. del Campo y M. Navarro, Análisis sociológico de la familia española, Min. Cultura, Madrid, 1982, págs. 114 y sigs. 
No puede ignorarse tampoco, partiendo de una perspectiva más abarcadora, la incidencia real en estas generaciones de planteamientos de signo radical y de otros modelos alternativos a la familia e inspirados en una determinada visión de la sociedad, la cultura, la relación hombre-mujer, la comunicación intergeneracional o la economía (experiencias comunales, movimientos de liberación de la mujer, formas de vida familiar inspiradas en los principios marxistas, propuestas críticas de los representantes de la antipsiquiatría contra la familia burguesa dominante, etc.).

Por razones obvias, nuestra atención se centrará preferentemente en los problemas relacionados con la política familiar. Partiendo de los artículos de la Constitución que establecen los principios básicos de toda normativa reguladora de los temas que afectan a la familia, existe un criterio fundamental para el análisis y valoración de las situaciones concretas que ha de afrontar el Trabajador social.

Más en particular, en nuestros CUADERNOS se irán examinando las modificaciones que se produzcan en nuestro derecho de familia para dar cumplimiento a lo que dispone el artículo 39 cuando afirma: «Los poderes públicos aseguran la protección social, económica y jurídica de la familia ${ }^{10}$. En este contexto se inscriben una serie de aspectos importantes relacionados, por ejemplo, con el trabajo, la seguridad social, la sanidad, la cultura, la atención a la tercera edad, la igualdad de oportunidades, la enseñanza y educación, la vivienda, la erradicación de situaciones marginales o la orientación profesional.

De acuerdo con nuestros planteamientos programáticos, se dará relevancia también a los problemas de los jóvenes. Por ejemplo, los que

10. Es significativo el hecho de que en el texto definitivo de este artículo la protección social aparezca antepuesta a la económica y política, que figuraban antes en los textos discutidos previamente. Como subrayaba I. Martín Martínez, «parece que se ha querido dar una primacía a lo social sobre toda clase de consideraciones» (La familia en la Constitución española de 1978, Rev. Fac. Der. Univ. Complutense, 1, 1978, pág. 43.

El desarrollo del concepto de protección social exige tener presentes otros artículos complementarios. El artículo 35, por ejemplo, llama la atención sobre la importancia del salario familiar. En el 47 se exige que los poderes públicos promuevan las condiciones necesarias y establezcan las normas pertinentes para hacer efectivo el derecho de todos a disfrutar de una vivienda digna. También se les pide en el artículo 49 la realización de una política de previsión, tratamiento, rehabilitación e integración de los disminuidos físicos, sensoriales y psíquicos. A la tercera edad se refiere el artículo 50 en el que se dice que deberá garantizarse, mediante pensiones adecuadas y periódicamente actualizadas, la suficiencia económica a los ciudadanos durante esta etapa de su vida, exigiendo además que se promueva su bienestar mediante un sistema de servicios sociales que atiendan sus problemas específicos. Estas y otras disposiciones similares se completan al referirse a la protección jurídica tendente a "promover las condiciones para que la libertad e igualdad del individuo y de los grupos en que se integra sean reales y efectivas» (art. 9.2). 
hacen referencia a situaciones concretas (personales, familiares, sociales, laborales...) que exigen nuestra participación e implicación en cuanto profesionales del Trabajo Social y de los Servicios Sociales y que se plantean constantemente en el ámbito de la vida cotidiana, ya sea en relación con la convivencia, el trabajo, la educación, la marginación o la delincuencia. Más allá, y como condición para afrontar con rigor esos problemas y poder ofrecer posibles alternativas o propuestas de actuación, nuestra atención se ampliará a cuestiones de carácter general que, directa o indirectamente, inciden en el fenómeno juvenil.

En este sentido, intentaremos ir ofreciendo una aproximación histórica rigurosa a los planteamientos que han venido haciéndose a propósito del cambio de valores, los procesos de identificación, los conflictos intergeneracionales, las subculturas juveniles o la disidencia crítica de los movimientos estudiantiles. Y ello se hará teniendo en cuenta los condicionamientos reales, manifiestos o latentes, que está a su base y la incidencia que tienen en ellos las transformaciones económico-políticas y los procesos socio-culturales que tienen lugar en nuestra sociedad compleja ${ }^{11}$.

Un ejemplo de esta orientación será la relevancia que iremos dando a los temas relacionados con la construcción de la identidad de los jóvenes, teniendo en cuenta las situaciones reales en que tienen lugar los procesos de comunicación, los modos de pertenencia a los diversos y cambiantes ámbitos de vida, la pluralidad de opciones que ofrece el sistema social, la posibilidad de manipulación de sus expectativas y el sentimiento de marginalidad, entre otros factores.

En este contexto tiene una especial significación la valoración positiva del llamado comportamiento anómico, entendido como un reflejo dinámico de la intensidad del cambio histórico. En otras palabras, se trata de ir más allá de la anomia entendida como factor destructivo o como manifestación de importancia, subrayando su significación como expresión de búsqueda y estímulo para el compromiso en la transformación de los modos de convivencia y de comportamiento, como una incitación para alumbrar nuevos valores y posibilidades inéditas de relación interhumana y de vida en común. En este sentido, es válida e incitadora la posición mantenida recientemente por Duvignaud que ve en ella un concepto muy apropiado para ayudarnos a definir una reflexión sociológica de las mutaciones y para precisar las posibilidades de intervención humana en la red de múltiples cambios que estamos viviendo ${ }^{12}$.

${ }^{11}$ G. Milanesi, I giovani nella società complessa, LDC, Torino, 1989; a propósito del tema concreto del trabajo y de los criterios que se proponen con motivo de la reforma que está en marcha en el sistema educativo, cf. M. Fernández Enguita, Educación, formación y empleo en el umbral de los noventa, CIDE, Madrid, 1990.

12 J. Duvigneaud, Ensayos sobre la anomia, Icaria Ed., Barcelona, 1990, pág. 42 y passim. En este contexto, sigue teniendo valor de obligada referencia el 
Desde otro punto de vista, interesa subrayar también la significación que tiene en los jóvenes contemporáneos la centralidad que otorgan a la libertad individual y a los valores de la subjetividad, como reacción frente al fenómeno de la nivelación que favorece y alienta nuestra sociedad. Este hecho explica, en parte, el predominio de la mentalidad utilitaria y de los criterios inmediatistas al afrontar problemas de la cultura, el trabajo o la convivencia. Partiendo de aquí, intentaremos una aproximación crítica a la nueva autocomprensión de los jóvenes y a las consecuencias que se derivan de ella en orden a entender sus posiciones cambiantes ante la familia, las instituciones escolares, las instancias económicas, las organizaciones, el sexo, la intercomunicación o la política, manteniéndose próximos a los marcos de comprensión que son propios de ellos.

Otra referencia importante de nuestros trabajos será, lógicamente, la atención constante a los problemas relacionados con la mujer, partiendo de las nuevas orientaciones y enfoques que tienden a ofrecer una visión positiva de su función en la comunidad. Como observa M. Nash, la revisión de los cánones interpretativos habituales de diversas disciplinas y campos de estudio ha significado un avance importante en el cuestionamiento de los sistemas de valores predominantes y ha hecho posible lo que ella denomina «reformulación de la conceptualización de la mujer» ${ }^{13}$.

Los nuevos planteamientos que han venido haciéndose a propósito de la mujer tienen su correspondencia en la forma de abordar el problema de su emancipación, que ha ido asumiendo un carácter más estructural y profundo. La igualdad real es buscada yendo más allá del tema-modelo de la complementariedad, pasando a un primer plano lo que D. Behnam caracteriza como «reivindicación de la propia identidad» ${ }^{14}$. Un ejemplo de esta nueva autoconciencia femenina se encuentra en la posición crítica que se mantiene ahora a propósito de la teoría de la terapia familiar que, en lo referente a los cambios profundos que han tenido lugar en la familia y a los roles de las mujeres den-

libro de Th. Roszak, El nacimiento de una contracultura, Kairós, Barcelona, 1976 (5. ${ }^{a}$ ed.) que veía en la contracultura la alternativa al mundo tecnocrático. En ella valoraba el profundo sentimiento de renovación y ese descontento radical que es susceptible de ir transformando nuestra civilización en algo que pueda ser identificado por ser humano como su hogar.

${ }^{13}$ Mary Nash, Conceptualización y desarrollo de los estudios en torno a las mujeres: un panorama internacional, Papers. núm. 30, 1988, pág. 19. La autora observa que el intento de comprensión de los mecanismos de opresión de las mujeres fue el eje interpretativo predominante en las décadas anteriores, mientras que ahora se intenta ir más allá contemplándolas también como puntos de referencia y de medida de lo humano.

${ }^{14}$ «Au-delà de l'égalité entre les sexes, il est question d'une revendication concernant l'identité propre» (D. Behnam, Une réflexion internationale sur l'avenir de la famille: un projet de l'UNESCO, Rev. Int. Sc. Soc., núm. 126, 1990, pág. 606). 
tro de ella, es considerada como rezagada con relación a la cultura en su totalidad. La nueva situación es resumida brevemente por M. Walters y P. Papp al caracterizar las diversas fases que pueden distinguirse en la evolución de la perspectiva feminista en la terapia familiar. Inicialmente se trataba de definir un marco de referencia feminista en común, pasando después a lo que denomina «fase de la exploración y la crítica» en la que al constatar la ausencia del género en las formulaciones de la teoría de los sistemas, fueron tomando conciencia de ciertos modelos sociales tendenciosos respecto a la función de los roles sexuales en las familias. En la fase actualmente en vigencia se trata de desarrollar intervenciones sistémicas no sexistas y feministas, revisando y adaptando los planteamientos sistémicos tradicionales e intentando crear pautas para introducir nuevas perspectivas en la práctica clíni$\mathrm{ca}^{15}$.

Uno de los fines de la nueva terapia familiar feminista consiste en la identificación del mensaje y las construcciones sociales basados en el género y que condicionan la conducta y los roles según el sexo. En este sentido, tiene importancia el reconocimiento de las limitaciones reales del acceso femenino a los recursos sociales y económicos y la toma de conciencia de un modo de pensar sexista que restringe las opciones de las mujeres de dirigir sus propias vidas. En la conclusión del estudio, las autoras subrayan que la esencia del trabajo clínico feminista radica en las actitudes del terapeuta hacia el «género» y en su sensibilidad a la repercusión diferencial de todas las intervenciones. Este es el principio, según ellas, que se abre paso a través de todas las metodologías y escuelas, pudiendo ser adaptado dentro de un amplio espectro de marcos teóricos y clínicos ${ }^{16}$.

Desde esta perspectiva de revalorización del protagonismo femenino se irán estudiando problemas concretos, sometiendo a un análisis crítico hechos y experiencias de la vida cotidiana y de la realidad social. En particular, nuestro empeño va a centrarse en la profundización de los nuevos planteamientos sobre los valores y conductas carac-

Desde otra perspectiva, es válida la contraposición que establece E. Gil Calvo entre la emancipación masculinista, que él describe como «insolidaria y depredatoria», irresponsable y unilateralmente instrumental, y la emancipación femenina que se busca y que ha de ser solidaria y estar «anclada en la relación expresiva con la alteridad» (La mujer cuarteada, Ed. Anagrama, Barcelona, 1991, págs. 213-214).

$15 \mathrm{M}$. Walters y Peggy Papp, Hacia una perspectiva feminista en la terapia familiar, en el vol. «la red invisible. Pautas vinculadas al género en las relaciones familiares», Paidós, Barcelona, 1991, págs. 29 y sigs.

16 IB., págs. 45-46; en este mismo volumen se contienen análisis y propuestas razonadas a propósito de las relaciones familiares (págs. 49 y sigs. y de problemas específicos como el divorcio, los hogares del progenitor único encabezados por mujeres, las familias resultantes de segundas nupcias o los modos de afrontar la situación de las mujeres solas, págs. 279 y sigs. 
terísticas de la mujer, deduciendo consecuencias y pautas de actuación para nuestra labor profesional.

$\mathrm{Al}$ abordar estos y otros problemas relacionados con la vida familiar, nuestra revista dará preferencia, según apuntamos ya anteriormente, a aquellos temas que tienen una singular relevancia para el desarrollo de nuestra labor profesional. Somos conscientes de la exigencia permanente de actualización de conocimientos y criterios que nos impone este compromiso con la realidad. Piénsese, por ejemplo, en la multiplicidad y complejidad de aspectos que inciden en la familia (personales y sociales, biológicos y psicológicos, económicos y políticos, terapéuticos y asistenciales) y que, de una forma u otra, inciden en nuestro trabajo. El título mismo de la revista («Alternativas»), quiere ser una invitación a esta ampliación de horizontes, a la búsqueda permanente, a la reflexión crítica, a la atención consciente a las situaciones y acontecimientos y, en suma, a hacer más eficaz y fecunda nuestra presencia en la sociedad. 